\title{
Development and Numerical Analysis of "Black-box" Counterpropagating Wave Algorithm for Exact Quantum Scattering Calculations
}

\author{
Bill Poirier \\ Department of Chemistry and Biochemistry, and Department of Physics, \\ Texas Tech University, Box 41061, Lubbock, Texas 79409-106档
}

\begin{abstract}
In a recent series of papers [J. Chem. Phys. 1214501 (2004), J. Chem. Phys. 124034115 (2006), J. Chem. Phys. 124034116 (2006)] a bipolar counter-propagating wave decomposition, $\Psi=\Psi_{+}+\Psi_{-}$, was presented for stationary bound states $\Psi$ of the one-dimensional Schrödinger equation, such that the components $\Psi_{ \pm}$approach their semiclassical WKB analogs in the large action limit. The corresponding bipolar quantum trajectories are classical-like and well-behaved, even when $\Psi$ has many nodes, or is wildly oscillatory. In this paper, the earlier results are used to construct a universal "black-box" algorithm, numerically robust, stable and efficient, for computing accurate scattering quantities of any quantum dynamical system in one degree of freedom.
\end{abstract}

\section{INTRODUCTION}

In a recent series of three articles,$\frac{1,2,3}{3}$ the use of bipolar counter-propagating wave methods $(\mathrm{CPWMs}) \frac{1,2,3,4,5}{1}$ for exact numerical solution of the time-independent Schrödinger equation was explored. The basic underlying idea is to decompose the stationary wavefunction, $\Psi$, into a sum of two counter-propagating wave components, i.e.

$$
\Psi=\Psi_{+}+\Psi_{-} .
$$

If done appropriately, such a decomposition can lead to very important ramifications for quantum trajectory methods (QTMs) 5,6,7.8,9,10,11,12,13,14 i.e., trajectorybased numerical techniques for performing exact quantum dynamics calculations, based on Bohmian mechanics $15,16,17,18,19,20$ due to nonlinearity of the Bohmian equations of motion. In particular, the earlier series of articles has culminated in a set of trajectorybased time-dependent methods for computing stationary scattering quantities (the theoretical underpinning of all chemical reactions) in one degree of freedom (DOF). These methods were found to be numerically stable and efficient for model systems within a certain regime of system parameters and accuracy typical of molecular

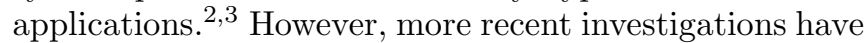
uncovered certain limitations that arise in more extreme cases. This has motivated the goal of the present paper, to perform a much more detailed numerical analysis of the methods, and to develop a single stable, robust, and efficient "black-box" algorithm that can be applied to virtually any 1 DOF system with a minimum of user intervention.

In recent years, QTM's have arisen as a very promising tool for performing accurate quantum dynamics calculations for many-DOF systems, using trajectory ensembles in a manner similar to classical simulations. ${ }^{21}$ In the conventional unipolar formulation of Bohmian mechanics, $\frac{15,16,17,18,19,20}{1}$ the MadelungBohm amplitude-phase decomposition of the 1 DOF $\Psi$, i.e.

$$
\Psi(x, t)=R(x, t) e^{i S(x, t) / \hbar},
$$

is used to generate the (presumably classical-like) quantum field functions, $R(x, t)$ and $S(x, t)$. For slowlyvarying potentials, the classical field functions are also slowly-varying, except in the vicinity of caustics. The corresponding quantum field functions of Eq. (2) behave similarly when there is no interference. However, interference introduces non-classical-like oscillations in the quantum field functions, which in turn lead to numerical difficulties for QTM calculations - collectively referred to as "the node problem" 5.10 (even though true nodes per se need not be present). As interference is formally unavoidable in any reactive scattering context, the node problem is to date the most important issue impeding the progress of QTM's as a general and robust tool for scattering dynamics applications, although much progress has been made, $\stackrel{4,22,23,24}{2}$

The bipolar CPWM approach to the node problem, as adopted here, and in the earlier series of articles,,$\frac{1,2,3}{, 2}$ is inspired by classical and semiclassical theories - which employ a multipolar, Eq. (1)-like decomposition as the means of preserving smooth and slowly-varying field functions. For stationary 1 DOF applications, the $\Psi_{+}$ and $\Psi_{-}$components are respectively, left- and righttraveling waves, with equal and opposite (group) velocities, $v(x)$ and $-v(x)$. Oscillatory interference behavior is not evident in the individual component field functions, but arises naturally from their linear superposition. For stationary bound state calculations, cancellation of flux requires that the component densities, $\rho_{+}(x)=\left|\Psi_{+}(x)\right|^{2}$ and $\rho_{-}(x)=\left|\Psi_{-}(x)\right|^{2}$, be equal. A reasonable generalization of classical field properties can be used to arrive at a unique exact quantum decomposition of the Eq. (11) form, which - unlike the conventional unipolar ansatz [Eq. (2)] - satisfies the correspondence principle in the large-action limit $\frac{1}{1}$

For stationary scattering, or continuum state calculations, the non-uniqueness and non-square-integrability of the quantum solutions complicate matters somewhat, $, 2,3$ 
as might be expected. Boundary conditions play an essential role, and specifically for the usual left-incident boundary conditions presumed here, necessarily imply asymmetry and non-zero flux for the corresponding exact quantum stationary solution. Conventional scattering theory already provides an asymptotic (i.e. $|x| \rightarrow$ $\infty)$ bipolar decomposition into the familiar "incident," "transmitted," and "reflected" plane wave componentsin fact the only decomposition possible that does not result in asymptotically oscillatory field functions. An exact quantum bipolar CPWM decomposition that respects all of the above can be constructed,,$\stackrel{2.3}{ }$ with $\Psi_{+}(x)$ joining the incident and transmitted waves continuously through the interaction region, and $\Psi_{-}(x)$, the reflected wave, damping to zero as $x \rightarrow \infty$. This is again achieved through analogy with semiclassical mechanics, albeit a "sophisticated" version. ${ }^{25,26,27}$ A key feature is that the trajectories are actually classical, with all quantum effects arising not from a quantum potential (as in conventional Bohmian mechanics), but through dynamical $\Psi_{ \pm}$ coupling. Conceptually, this gives rise to instantaneous trajectory hopping ${ }^{28}$ from one CPWM component to the other, in the interaction region where the potential $V(x)$ is changing. In effect, one has a local, time-dependent theory of stationary scattering, instead of the more traditional global, time-independent picture.

The new theory thus provides some pedagogical insight, analogous to ray optics and cavity ring-down experiments, 2,29 However, this paper focuses primarily on numerical aspects of the new approach-which may be regarded as a relaxation method with exponentiallyfast convergence, for computing stationary scattering states of desired boundary conditions, and associated reactive scattering quantities such as reflection and transmission probabilities. In comparison with other quantum scattering methods, the bipolar CPWM approach offers some decided numerical advantages. In particular, there is no need to invoke complex scaling (analytical continuation $)^{30,31,32}$ or absorbing boundary conditions (optical potentials) $\stackrel{33,34,35,36,37,38,39}{,}$ as trajectories and field quantities are real-valued throughout. Moreover, the scaling of computational (CPU) effort is linear with the grid size, $N$, rather than proportional to $N^{3}$.

The new approach may therefore lead to some of the most efficient exact quantum scattering algorithms in existence, but certain practical limitations must first be overcome. To begin with, the previous papers propose several distinct algorithms; a detailed numerical analysis must be performed to determine which of these is "best," from the perspective of numerical stability, robustness, and efficiency. In particular, some of these algorithms appear to be ineffective beyond a certain desired level of accuracy (Sec. IIB). The reasons for this must be elucidated via systematic study, in order to develop more efficient implementations. An even greater motivation, though, is the fact that the above problem is greatly exaggerated in the multidimensional case, resulting in numerical instabilities that have thus far hindered efforts in this direction. Another limitation is that some of the previous algorithms are not applicable to completely general systems (Sec. IIA). This is addressed here by generalizing the methodology to allow for a nearly arbitrary choice of "classical" trajectory (Sec. IIID). Finally, the robustness of the new algorithms must be demonstrated by application to representative molecular systems encompassing a very broad range of potentials, system parameters, and desired accuracy levels. One such application area, known to cause difficulties for conventional semiclassical and exact quantum methods, is particularly importanti.e., the deep tunneling regime (Sec. IVB). Other examples considered here include asymmetric potentials with barriers (Sec. IVD) and systems with reaction intermediates (Sec. IVE).

\section{BACKGROUND}

\section{A. Exact Quantum Dynamics Using Counterpropagating Trajectories}

Here, we summarize some of the developments of Refs. 1-3. The basic goal is to define a set of timeevolution equations for the two 1 DOF counterpropagating waves, $\Psi_{ \pm}(x, t)$, such that in the large $t$ limit, the total $\Psi(x, t)$ of Eq. (11) approaches the exact stationary state evolution for the desired energy, $E$, and boundary conditions. This general approach is thus a "relaxation method," for which $\Psi(x, t)$ at intermediate times need not adhere to the actual time-dependent Schrödinger equation - although there are versions of the method for which the latter property is also satisfied in a sense. $\stackrel{2.3}{.}$ For the usual left-incident boundary condition, the initial reflected wave must be zero, i.e. $\Psi_{-}(x, 0)=0$. The initial $\Psi_{+}(x, 0)$ is essentially arbitrary, as it is presumed 2.3 that any reasonable choice will converge exponentially quickly in the large time limit. However, two natural choices have been considered: (1) $\Psi_{+}(x, 0)=\exp [i \sqrt{2 m E} x / \hbar]$, i.e. incident asymptotic plane wave extended throughout space [with $V(x) \rightarrow 0$ presumed as $x \rightarrow-\infty$ ]; (2) $\Psi_{+}(x, 0)=\Theta\left(x_{L}-x\right) \exp [i \sqrt{2 m E} x / \hbar]$, i.e. incident wave truncated at left edge of interaction region, $x_{L} \leq x \leq x_{R}$. In Ref. 3, (1) and (2) above are referred to respectively as "non-wave-front" and "wavefront" versions. The latter gives rise to a useful cavity ring-down dynamical interpretation,,$\stackrel{2,29}{,}$ although the former is found to converge more quickly to the exact solution, and is therefore used throughout this paper. Note that (1) also has its own interpretation, to be discussed shortly.

From Ref. 3, we also learn that there are two distinct semiclassical formalisms that can be applied to generate exact Eq. (1) decompositions (and associated time-evolution equations) with the desired theoretical properties - the Bremmer approach $(\mathrm{B})^{27}$ and the Fröman approach $(\mathrm{F}) \stackrel{26}{\underline{2}}$ Of the two, the B approach can 
only be applied when the trajectories are classical trajectories. Unfortunately, classical trajectories cause numerical instabilities for both $\mathrm{B}$ and $\mathrm{F}$ evolutions whenever there is tunneling, i.e. for all below-barrier energies, $E$, and is therefore not a viable choice for a completely "robust" algorithm. The F approach, on the other hand, can be generalized for almost any desired choice of "semiclassical" trajectory. In particular, constant velocity trajectories (where the speed corresponds to the incident plane wave energy $E$ ) handle tunneling without difficulty, and lead to smoother field functions and remarkably simple F time-evolution equations:

$$
\frac{d \Psi_{ \pm}}{d t}=\frac{i}{\hbar}(E-V) \Psi_{ \pm}-\frac{i}{\hbar} V \Psi_{\mp}
$$

Some comments on Eq. (3) are in order. First, these are the Lagrangian, or "hydrodynamic" equations of motion, meaning that the left hand-side is a total time derivative, as would be appropriate for a trajectory (moving-grid) calculation. Note that they require no differentiation of any kind - not even of the potential itself, to construct force fields. Note also that the final, coupling term, i.e. that responsible for all quantum corrections beyond the "semiclassical" approximation, is proportional to $V(x)$, and vanishes in the $x \rightarrow-\infty$ reactant asymptotic limit. It also vanishes in the product asymptote provided the potential is "asymptotically symmetric," i.e. $V(x) \rightarrow=0$ as $x \rightarrow \infty$ (the asymmetric case will be addressed in Sec. IIIC). This ensures that the $\Psi_{ \pm}(x)$ solutions exhibit the requisite plane wave behavior for the desired energy $E$. Finally, we note that for the special case $V=0$, i.e. the potential that would classically give rise to the constant velocity trajectories used, all coupling vanishes. This implies that the nonwave-front initial condition (1) described above is in fact the "basic WKB" 3,26 approximation in the generalized F sense, as will be exploited in Sec. IIID.

Since the $\Psi_{+}$and $\Psi_{-}$trajectories move in opposite directions, the two moving grids are necessarily incommensurate at an arbitrary time, which numerically necessitates the use of interpolation for evaluating the coupling term in Eq. (3). Alternatively, an "Eulerian" or fixed-grid implementation can be adopted, for which the corresponding time-evolution equations are

$$
\frac{\partial \Psi_{ \pm}}{\partial t}=\mp v \Psi_{ \pm}^{\prime}+\frac{i}{\hbar}(E-V) \Psi_{ \pm}-\frac{i}{\hbar} V \Psi_{\mp},
$$

where $v=\sqrt{2 E / m}$ is the trajectory speed, and primes denote spatial differentiation. The grids are now commensurate at all times, obviating the need for interpolation, but numerical differentiation is now required to evaluate the new convective term in Eq. (4).

\section{B. Numerical Analysis of Fixed-grid Scheme}

Of the various possible numerical schemes presented in Ref. 3 and above, we have thus far settled on just two can- didates for the present purpose: the trajectory and fixedgrid versions of the non-wave-front, $\mathrm{F}$, constant-velocity trajectory method. To choose one over the other, we must consider the stated criteria of numerical stability, efficiency, and robustness (i.e. generality of applicability across different systems and computational accuracy levels).

In Ref. 3, all of the calculations were applied to one degree-of-freedom (1 DOF) systems with similar parameter values and energies, and for the most part, to a desired accuracy level of $10^{-4}$. In this context, the two numerical schemes were found to be of roughly comparable efficiency (i.e. total CPU time), with the trajectory approach requiring substantially fewer grid points (around $4 \times$ per DOF) and larger time steps $(\times 10)$, but a much longer CPU time per step, due primarily to the nearest-neighbor search algorithm that was employed. On the other hand, there were also clear indications that the trajectory method would become superior at higher accuracies - e.g., Ref. 3 Fig. 5, which suggests that the fixed-grid error for reflection and transmission probabilities cannot be reduced below $10^{-5}$ or so, no matter how many grid points are used.

A limit on achievable accuracy is clearly not a desirable trait for a robust method, and in particular, is complete anathema for accurate deep tunneling applications. Consequently, a detailed numerical analysis of this situation was performed for this paper, in order to gain a thorough understanding of the factors responsible. In the course of this, it was discovered that the limiting accuracy can be substantially worse for other reasonable system parameter choices, especially for multidimensional systems, and/or those with reaction intermediates - lending additional imperative to the exercise. The manifestation of the error was quickly identified as oscillations in the right-asymptotic density $\rho_{+}(x)=\left|\Psi_{+}(x)\right|^{2}$, which form around $x=x_{R}$, and then propagate inward over timewhereas it is clear from Eq. (3) that the solution $\rho_{+}^{\prime}(x)$ should be zero asymptotically.

Ref. 3, and the above-mentioned preliminary numerical investigations for this paper, computed fixed-grid spatial derivatives using centered, fourth-order finite differences $\underline{40}$ (one-sided for the endpoints $x_{R}$ and $x_{L}$ ), and fourth-order Runge-Kutta time integration $\stackrel{41}{\underline{11}}$ However, the bulk of the present analysis uses second-order finite difference (two-sided for interior grid points and onesided for endpoints) and first-order forward Euler time integration, as a deliberate means of exacerbating the problem and simplifying the analysis, in order to identify the cause. The oscillation amplitudes were indeed found to be much larger in this case. It was also found that the oscillation wavelength was always 2-3 grid points, regardless of the density of grid points used. With respect to number of grid points, $N$, (keeping $x_{L}$ and $x_{R}$ fixed), the amplitude oscillation was found to decrease roughly inversely. Reducing the time step had no effect whatsoever on the oscillation amplitude or wavelength, but did reduce the inward propagation speed, roughly pro- 
portionally.

All of the above findings support the notion that the oscillation problem is somehow due to spatial differentiation. It might also be theorized that oscillations arise from the $\Psi_{ \pm}$phase difference inherent in the coupling term of Eq. (3), but the wavelengths are incommensurate with what is actually observed. To definitively rule out coupling as the source of the problem, however, a calculation was performed for the $V=0$ free particle system, for which there is no coupling - yet the above oscillations were still observed. Another alternative explanation would be von-Neumann instabilities $\underline{42}$ A straightforward theoretical analysis is possible for the free particle case, and so this was conducted, and compared to numerical tests for a wide range of time steps and grid spacings. In the theoretically von Neumann stable regime, the oscillations were still observed, whereas in the von Neumann unstable regime, the numerical solutions diverged over time as expected - a different phenomenon entirely.

In fact, none of the vast literature on PDE simulations of which the author is aware can account for the odd oscillatory behavior observed. The only sensible explanation of the cause can therefore be the "mixed" boundary conditions, which are highly non-standard, and perhaps have not been considered previously. In particular,

$$
\Psi_{-}\left(x_{R}, t\right)=0 \quad ; \quad \Psi_{+}\left(x_{L}, t\right)=\exp \left[-\frac{i}{\hbar} E t\right],
$$

so that the two different components have boundary conditions on opposite sides. This situation is completely natural, given the counterpropagating nature of the method, and cannot be changed through any straightforward theoretical recasting. Within the fixed-grid framework, various numerical solutions have been essayed, including asymptotic plane wave rescaling, and amplitude/phase decomposition of the endpoint $\Psi_{ \pm}$components, but none appears to provide substantial relief from the oscillation problem described above.

\section{THEORY AND ALGORITHM DEVELOPMENT}

\section{A. Constant-velocity Trajectory Scheme}

We are thus led naturally to the trajectory, rather than fixed-grid, version of the non-wave-front, F, constantvelocity method. Ref. 3 Fig. 5 indicates the high accuracies (around $10^{-8}$ ) of which the trajectory approach is capable. This can be largely attributed to the fact that the trajectory version does not require numerical spatial differentiation. However, a more detailed numerical analysis reveals that the errors in this case also manifest as asymptotic oscillations, albeit of much smaller amplitude than the fixed-grid case, and with different scaling. More specifically, for a test Eckart system comparable to that of Ref. 3, an inverse quartic relation is found between the oscillation amplitude and grid size, $N$. This can also be roughly inferred from Ref. 3 Fig. 5 , and is in any case not surprising, given that third-order interpolation is used (Sec. IIIB).

As in the fixed-grid case, the oscillation amplitudes and wavelengths are completely unaffected by time-step size. However, the latter quantity does turn out to be closely related to another, more serious type of numerical error also observed: in the large $t$ limit, the magnitude of $\Psi_{+}(x, t)$ grows linearly with $t$ in the left-asymptotic region. For typical $\Delta$ values and desired accuracy levels, using fourth-order time evolution as in Ref. 3 , the growth rate is so slow as to contribute insignificantly to the final error. However, this asymptotic growth does present a very significant challenge for extremely accurate calculations (e.g. $10^{-12}$ as in Sec. IVB) and must therefore be addressed. Once again, to exaggerate the effect for analytical purposes, first-order forward Euler time integration was used. For the test Eckart system, the growth rate (with respect to system time, not number of time steps) was found to depend roughly linearly on $\Delta$, and for typical Ref. 3 values ( $\Delta=3.0$ a.u., $t_{\max }=6000$ a.u.) resulted in a final error of $10 \%$.

An effective solution to the asymptotic growth problem has been found, but first it is necessary to divulge further details of the trajectory implementation, as presented schematically in Fig. 1. At $t=0$, the two trajectory grids, i.e. "upper" (for $\Psi_{+}$) and "lower" (for $\Psi_{-}$) are coincident, with grid points at both $x_{L}$ and $x_{R}$, and a uniform grid spacing of $\Delta x=\left(x_{R}-x_{L}\right) /(N-1)$. Over time, $x_{N}^{+}$, the right-most trajectory of the upper grid, evolves beyond the interaction region, as does $x_{1}^{-}$, the left-most point of the lower grid. At time $t_{\text {shift }}=\Delta x / v$, when these extremal points have progressed one grid spacing beyond the interaction region, they are discarded, and new upper/lower grid trajectories are introduced at $x_{L} / x_{R}$, and assigned $\Psi_{ \pm}$values in accord with Eq. (5). The indexing for both grids is shifted by one, resulting once again in coincident grids identical to that at $t=0$. This approach obviates the requirement of a nearest-neighbor search algorithm, thus greatly reducing CPU cost per time step. It does require that $t_{\text {shift }}$ be a multiple of $\Delta$, although in practice, this poses a very minor constraint.

Note from Fig. 1 that at general times within the cycle described above, one or two points from each grid lie outside the range of the opposite grid. In such cases, one might consider resorting to extrapolation to evaluate the coupling term in Eq. (3), although this approach is not recommended $\underline{\underline{42}}$ Instead, since the exterior points all lie near the edges of the interaction region, we simply set $V \rightarrow 0$ in Eq. (3). In principle, this results in unitary plane wave propagation (PWP) for the exterior points. In practice, the Euler time integrator used here is not unitary (nor is Runge-Kutta) - thus causing the observed growth of asymptotic probability. This situation is well understood in the literature, and various alternative unitary time integrators exist $\underline{\underline{42}}$ However, since the analytical plane wave solution is known, we simply adopt 


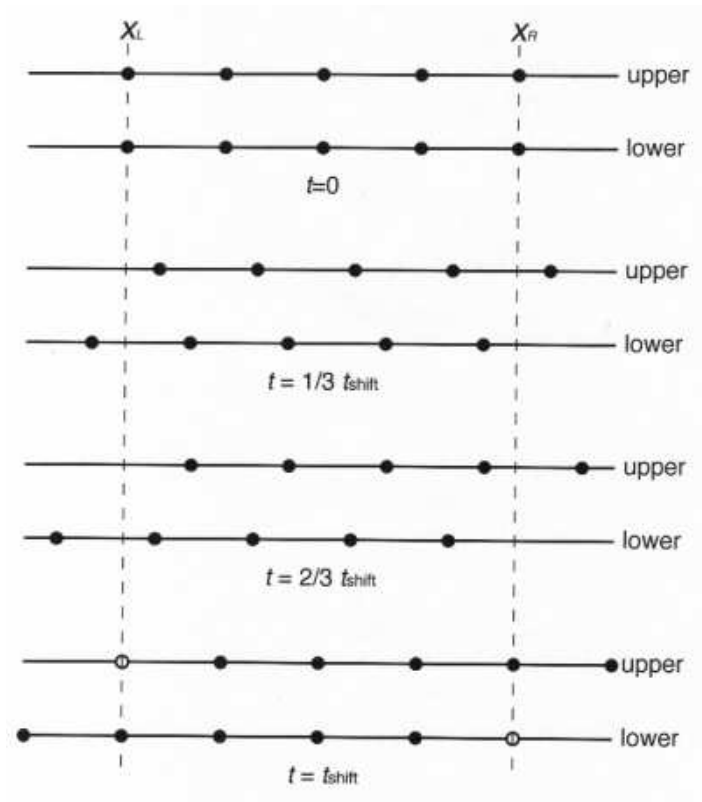

FIG. 1: Schematic indicating location of trajectory grid points (circles) over time, for upper $\left(\Psi_{+}\right)$and lower $\left(\Psi_{-}\right)$grids of the constant velocity trajectory bipolar counterpropagating wave method. At $t=0$, the $N=5$ points of both grids are distributed uniformly from $x_{L}$ to $x_{R}$, with grid spacing $\Delta x=$ $\left(x_{R}-x_{L}\right) /(N-1)$. Over time, the upper/lower grid points move with constant velocity $\pm v= \pm \sqrt{2 E / m}$. At time $t_{\text {shift }}=$ $\Delta x / v$, the two grids are again coincident in the interior region. The two outermost points are deleted, and replaced with two new points (indicated with open circles) at the opposite ends of their respective grids, to restore the situation at $t=0$.

the following exact unitary PWP scheme:

$$
\Psi_{ \pm k}(t+\Delta)=\Psi_{ \pm k}(t) \exp \left[\frac{i}{\hbar} E \Delta\right]
$$

where $1 \leq k \leq N$ indexes individual trajectories.

The above PWP modification, applied to extreme points on both sides of the two grids in lieu of Euler time integration, completely eradicates the asymptotic growth of probability over time. However, it leads to a new problem, i.e. asymptotic "ramping." The idea here is that as the $x_{1}^{+}$trajectory travels inward, calculation of the plane wave portion of Eq. (3) shifts suddenly from the unitary PWP to the nonunitary Euler integrator, causing the final converged solution to manifest a gradual increase in asymptotic $\rho_{+}(x)$ from left to right. This spurious ramping was found to be on the order of a few percent for the test Eckart system - though the effect is once again greatly exaggerated here, due to the first-order time integrator used. Still, even with realistic time integrators (e.g. fourth-order Runge Kutta) the ramping problem would cause difficulties for very highaccuracy calculations. A simple solution is nevertheless available: use the Eq. (6) PWP for the plane wave portion of Eq. (3) throughout the coordinate range (i.e. not just in the asymptotic extremes). The first-order forward implementation is therefore as follows:

$$
\begin{aligned}
\Psi_{ \pm k}(t+\Delta) & =\Psi_{ \pm k}(t) \exp \left[\frac{i}{\hbar} E \Delta\right] \\
& -\frac{i}{\hbar} V\left(x_{k}^{ \pm}\right) \Delta\left[\Psi_{ \pm k}(t)+\Psi_{\mp}\left(x_{k}^{ \pm}, t\right)\right]
\end{aligned}
$$

where $\Psi_{\mp}\left(x_{k}^{ \pm}, t\right)$ is computed via interpolation. Use of Eq. (7) has eradicated the nonunitary error completely in the test Eckart application with parameters described above, resulting in a numerical solution accurate to several parts per thousand throughout the full $x$ range. The error that remains adheres to the form of interpolation error, as discussed above.

\section{B. Interpolation}

As interpolation is a primary source of numerical error, it is essential for the present purpose that this operation be performed in as efficient and accurate a manner as possible. One of the key presumptions of successful interpolation is that the underlying mathematical function be smooth and well-behaved,$\frac{5,42}{5}$ This results not only in more accurate computation, but also better characterization of the numerical analysis. Fortunately, the bipolar CPWM methodology is designed to provide smooth, slowly-varying field functions even when $\Psi$ itself is not; these have indeed been observed in all cases considered thus far, with the $\mathrm{F}$ constant-velocity functions being particularly slowly-varying. Other desired attributes of the interpolation routine include generality, robustness, systematic error reduction, and a minimum of required user intervention ${ }^{\underline{5}}$

In the previous work,$\frac{3}{,}$ fourth-order local polynomial interpolation was used, analogous to a moving least squares method with a stencil of five grid points for the interior of the grid $\stackrel{5.42}{ }$ At the grid edges, fewer stencil points (and lower-order polynomials) were used. In principle, if the field functions are sufficiently smooth, and the grid points ideally distributed, then higher-order calculations can lead to more accurate results. However, this is not guaranteed, and in some cases higher-order interpolation may introduce unwanted wild oscillation be-

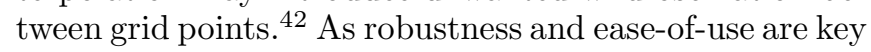
for the present purpose as described above, we simply adopt third-order interpolation as the most reasonable (and standard) choice. Also, since edge effects appear to be so important (Sec. IIB), we retain third-order interpolation to the edges of the grids, i.e. four-point stencils are used throughout for polynomial interpolation. We also consider spline interpolation, $\stackrel{42}{,}$ for which the "stencil" is always two grid points, in effect, regardless of order. Spline interpolation has the advantage of continuous second derivatives throughout the coordinate range, yielding "stiffer" interpolants that are more stable, and generally more accurate for smooth functions.

The numerical implementation described in Sec. IIIA employs interpolation only when evaluating the coupling 
term, i.e. the very last $\Psi_{\mp}\left(x_{k}^{ \pm}, t\right)$ quantity in Eq. (7). Although Eqs. (6) and (77) are written in terms of $\Psi_{ \pm}$, in fact it is the real-valued field quantities, $r_{ \pm}$and $s_{ \pm}$, obtained from

$$
\Psi_{ \pm}(x)=r_{ \pm}(x) e^{i s_{ \pm}(x) / \hbar}
$$

that are designed to be most smooth and slowly-varying (especially in the classical limit) $1,2,2,3$ Numerically, one might consider storing $r_{ \pm k}$ and $s_{ \pm k}$ values instead of $\Psi_{ \pm k}$ 's, but the corresponding Eq. (77) becomes complicated, nonlinear, and numerically less stable.
Instead, we adopt a "best of both worlds" approach, whereby $\Psi_{ \pm k}$ values are stored and used in Eqs. (6) and (77), $r_{\mp k}$ 's and $s_{\mp k}$ 's obtained at each time-step, and the latter interpolated to compute the coupling term in Eq. (7) via Eq. (8). Obtaining $r_{\mp k}=\left|\Psi_{\mp k}\right|$ is straightforward; however, $s_{\mp k}=\hbar \arctan \left(\operatorname{Im} \Psi_{\mp k} / \operatorname{Re} \Psi_{\mp k}\right)$ requires special care in the choice of branch cut, to ensure continuity of the field function throughout the coordinate range. Our simple solution to this well-known dilemma ${ }^{5}$ is the recursive implementation

$$
s_{\mp(k+1)}= \begin{cases}s_{\mp k} & \text { for } \Psi_{\mp(k+1)}=0 ; \\ \hbar \arctan \left[\frac{\operatorname{Im} \Psi_{\mp(k+1)}}{\operatorname{Re} \Psi_{\mp(k+1)}}\right] & \text { for } \Psi_{\mp k}=0 ; \\ s_{\mp k}+\hbar \arctan \left[\frac{\operatorname{Im}\left(\Psi_{\mp(k+1)} / \Psi_{\mp k}\right)}{\operatorname{Re}\left(\Psi_{\mp(k+1)} / \Psi_{\mp k}\right)}\right] & \text { otherwise, }\end{cases}
$$

with $\Psi_{\mp 0}=0$ by definition. Note also that due to the regular structure of the grid, there is no need for a search algorithm to compute the stencil for a given interpolant point, i.e. the total CPU effort per time-step associated with interpolation scales as $N$ rather than $N \log N$, placing it on a par with potential function evaluation.

The above $r / s$ interpolation scheme yields phenomenal improvements in accuracy when applied to the test Eckart problem of Sec. IIIA as compared to the $\Psi_{ \pm}$interpolation applied there. The numerical solutions still exhibit asymptotic oscillations; however, these are no longer constant in amplitude, but damp exponentially with increasing $|x|$, as do the true mathematical solutions. In any case, at the $-x_{L}=x_{R}=3.0$ a.u. grid-edge values considered, the amplitude has been reduced from a few parts per thousand (Sec. IIIA) to a few parts per million, using a grid of only $N=31$ points.

We conclude this subsection with a brief numerical comparison between polynomial and spline interpolation (both third order) in the context of the above algorithm as applied to the Eckart B problem with $E=$ $0.40 V_{0}$ (Sec. IVA). First though, as any complete spline implementation requires specification of the boundary conditions, we compared the accuracy of zero-secondderivative boundaries (natural splines) vs. the more usual zero-first-derivative case, for known functions similar to those computed below. The natural spline case was found to be about an order of magnitude more accurate throughout the coordinate range, and is therefore adopted.

For comparative purposes, both natural spline and polynomial interpolation were applied to the Eckart B problem. Both cases are characterized by quick convergence with respect to grid size, $N$, with the spline case converging more quickly. With respect to the time step,
$\Delta$, polynomial interpolation converges more quickly at first-i.e., for larger $\Delta$ values and lower accuracies. Beyond a certain point however, the polynomial error flattens out and even increases with subsequent decrease in $\Delta$ (even if $N$ is allowed to increase), whereas the spline error becomes arbitrarily small. Further analysis reveals that this behavior of the polynomial interpolation is due to asympotic oscillations, which increase in amplitude with decreasing $\Delta$, whereas in the spline case, the oscillation amplitudes are independent of $\Delta$. The polynomial case thus exhibits an effective minimum achievable error, on the order of $10^{-5}$ for the case considered (note: in conjunction with first-order forward time evolution). The parameters used for this optimized polynomial calculation were also applied to a spline calculation, which was found to be both more accurate (orders of magnitude so for the transmission probability) and faster (by about $30 \%$ ). Taking these facts into consideration, we adopt natural cubic spline interpolation from here on out.

\section{Discontinuous Constant Velocity Scheme}

The constant-velocity trajectory method as discussed above is not applicable for asymptotically asymmetric potentials ${ }^{3}$ i.e., $V_{L}=0 \neq V_{R}$, where $V_{L}=$ $\lim _{x \rightarrow-\infty} V(x)$ and $V_{R}=\lim _{x \rightarrow \infty} V(x)$. Mathematically, this is because the coupling term in Eq. (3) does not vanish in the product asymptote $(x \rightarrow \infty)$, and so the $\Psi_{ \pm}(x, t)$ components do not converge over time. Physically, it is because the trajectory velocity $v$ is not commensurate with the product translational kinetic energy, $\left(E-V_{R}\right)$. In any event, the presumption $V_{L}=V_{R}$ is too restrictive in practice to be of general use, and so some appropriate resolution must be found. One might 
consider the classical trajectory bipolar CPWM, for instance, which would work fine for barrierless reactions; however, the more general and realistic case of an asymmetric reaction with a barrier (Fig. 3) - and therefore below-barrier energies - would seem to be beyond the reach of either method.

On the other hand, the above difficulty is simply a manifestation of a standard concern that is not at all new in reactive scattering theory-i.e., that the product and reactant asymptotic potentials, and associated quantum states, are not the same $\stackrel{43}{ }^{\text {Various remedies }}$ have been prescribed for this, including use of a "dividing surface" 34.35 that partitions configuration space into reactant and product sides. For 1 DOF applications, the dividing "surface" is just a single point, labelled $x_{0}$. We can easily incorporate the dividing point idea into the constant velocity scheme by using trajectories with reactant asymptotic velocities in the $x<x_{0}$ region, and another set with product asymptotic velocities in the $x>x_{0}$ region. The appropriate generalization of Eq. (3) (which holds for any asymptotic values of the potential) is

$$
\begin{aligned}
\frac{d \Psi_{L / R \pm}}{d t}= & \frac{i}{\hbar}\left(E-V-V_{L / R}\right) \Psi_{L / R \pm} \\
& -\frac{i}{\hbar}\left(V-V_{L / R}\right) \Psi_{L / R \mp},
\end{aligned}
$$

with " $L$ " referring to the reactant (left) side of $x_{0}$, and " $R$ " the product (right) side. The corresponding trajectory velocities are $v_{L / R}=\sqrt{2\left(E-V_{L / R}\right) / m}$.

Although $\Psi$ itself, and its first spatial derivative, must be continuous across $x=x_{0}$ at all times, the components $\Psi_{ \pm}$are decidedly discontinuous. In a trajectory implementation, $\Psi_{L+}\left(x_{0}\right)$ and $\Psi_{R-}\left(x_{0}\right)$ are known quantities, and $\Psi_{L-}\left(x_{0}\right)$ and $\Psi_{R+}\left(x_{0}\right)$ are unknown; however, the $\Psi$ constraint above can be used to uniquely specify the

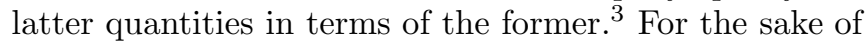
numerical efficiency, it is advisable that $\Psi_{L+}$ and $\Psi_{R-}$ trajectories cross $x_{0}$ at exactly the same time; when this occurs, these "edge" trajectories (for their respective regions) are destroyed, and new edge trajectories for $\Psi_{L-}$ and $\Psi_{R+}$ simultaneously created without needing to resort to extrapolation. This is also when trajectory "shifting" occurs, as per Sec. IIIA. The above trajectory crossing condition requires that the grid spacings be different on either side of $x_{0}$, i.e. $\Delta x_{L / R}=t_{\text {shift }} v_{L / R}$. In other respects, the algorithm is similar to Sec. IIIA except that when $x_{k} \neq x_{0}$, we can not use Eq. (6) for the edge trajectories near $x_{0}$, because the coupling in this region is significant. Instead, we must use Eq. (7), even though this implies extrapolation within a range $\left(\Delta x_{L / R}\right) / 2$ of $x_{0}$.

\section{Ramp Trajectory Scheme}

It would be beneficial to develop a single, robust, and efficient bipolar CPWM scheme with none of the limitations of the previously-described methods. The method should in other words be continuous, slowly-varying and extrapolation-free, yet applicable to completely general potentials such as that of Fig. 3. From the discussion in Sec. IIA, it is natural to consider the generalized F approach in this regard. Specifically, the "semiclassical" trajectories considered need have no direct relation to the actual potential $V(x)$, but rather, are defined for some arbitrary effective potential, $V_{\text {eff }}(x)$, as $v(x)=$ $\sqrt{2\left[E-V_{\text {eff }}(x)\right] / m}$. The specific choice $V_{\text {eff }}(x)=V(x)$ reproduces the classical trajectory scheme, $V_{\text {eff }}(x)=0$ the constant-velocity scheme, and $V_{\text {eff }}(x)=V_{L}+\left(V_{R}-\right.$ $\left.V_{L}\right) \Theta\left(x-x_{0}\right)$ the discontinuous scheme.

The ideal $V_{\text {eff }}(x)$ should:

1. approach the flat $V(x)$ function in both asymptotic limits, i.e. $\lim _{x \rightarrow \mp \infty} V_{\text {eff }}(x)=V_{L / R}$

2 . vary smoothly and monotonically through the interaction region.

Condition 1. results in vanishing asymptotic coupling, whereas 2. ensures there are no turning points, so that barrier tunneling poses no difficulties. A sigmoid ramp function is thus clearly indicated; below, we present a specific form based on the tanh function that is applicable to extremely general situations. Although this form might not be ideally suited to systems with reaction intermediates, one could in that case add several ramp potentials together to match the reaction profile appropriately. In any event, we must first work out the theory and numerical implementation for the generalized Fbased CPWM approach.

The generalized F decomposition of Eq. (11) for stationary scattering states in 1 DOF is uniquely determined from the time-independent Schrödinger equation and the relation ${ }^{3,26}$

$$
\Psi^{\prime}=-\frac{v^{\prime}}{2 v} \Psi+\frac{i m v}{\hbar}\left(\Psi_{+}-\Psi_{-}\right) .
$$

For completely general $v(x)$, these lead to

$$
\Psi_{ \pm}^{\prime}=\left[-\frac{1}{2}\left(\frac{v^{\prime}}{v}\right) \pm \frac{i}{\hbar} m v \pm \frac{i}{2} A\right] \Psi_{ \pm} \pm \frac{i}{2} A \Psi_{\mp},
$$

where

$$
A=\left(\frac{\hbar}{m v}\right)\left[\frac{3}{4}\left(\frac{v^{\prime}}{v}\right)^{2}-\frac{1}{2}\left(\frac{v^{\prime \prime}}{v}\right)-\frac{2 m}{\hbar^{2}}\left(V-V_{\mathrm{eff}}\right)\right] .
$$

For consistency, it is convenient to reexpress the $v$ derivatives above in terms of $V_{\text {eff }}$ and its derivatives. If the trajectory equations of motion are to have no $\Psi_{ \pm}$ spatial derivatives as desired, then we must construct a convective term somehow; this is easily accomplished by multiplying Eq. (12) by $\pm v$. The result is:

$$
\begin{aligned}
\pm v \Psi_{ \pm}^{\prime}= & {\left[ \pm \frac{1}{4} v\left(\frac{V_{\mathrm{eff}}^{\prime}}{E-V_{\mathrm{eff}}}\right)+\frac{i}{\hbar}\left(m v^{2}-V+V_{\mathrm{eff}}+C\right)\right] } \\
& \times \Psi_{ \pm}-\frac{i}{\hbar}\left[V-V_{\mathrm{eff}}-C\right] \Psi_{\mp}, \quad \text { where }
\end{aligned}
$$




$$
C=\left(\frac{\hbar^{2}}{2 m}\right)\left[\frac{5}{16}\left(\frac{V_{\mathrm{eff}}^{\prime}}{E-V_{\mathrm{eff}}}\right)^{2}+\frac{1}{4}\left(\frac{V_{\mathrm{eff}}^{\prime \prime}}{E-V_{\mathrm{eff}}}\right)\right]
$$

Next, we replace $m v^{2}$ in Eq. (14) with $2\left(E-V_{\text {eff }}\right)$. Adding $-(i / \hbar) E \Psi_{ \pm} \mp v \Psi_{ \pm}^{\prime}$ to both sides, and recognizing that for the stationary solution, $\partial \Psi_{ \pm} / \partial t=-(i / \hbar) E \Psi_{ \pm}$, we obtain finally

$$
\begin{aligned}
\frac{\partial \Psi_{ \pm}}{\partial t}= & \mp v \Psi_{ \pm}^{\prime} \\
& +\left[ \pm \frac{1}{4} v\left(\frac{V_{\mathrm{eff}}^{\prime}}{E-V_{\mathrm{eff}}}\right)+\frac{i}{\hbar}\left(E-V-V_{\mathrm{eff}}+C\right)\right] \Psi_{ \pm} \\
& \quad-\frac{i}{\hbar}\left[V-V_{\mathrm{eff}}-C\right] \Psi_{\mp}
\end{aligned}
$$

Note that for constant velocity trajectories, $C=V_{\text {eff }}=$ 0, and Eq. (16) reduces to Eq. (4), as it should. Also interesting (and useful) is the fact that Eq. (16) satisfies the combined continuity relation ${ }^{3}$-i.e. the total combined probability $\left(\rho_{+}+\rho_{-}\right)$is conserved over time, even though the individual $\rho_{ \pm}$are not. The precise flux relation giving rise to this is

$$
\frac{\partial \rho_{ \pm}}{\partial t}=-j_{ \pm}^{\prime} \pm \frac{2}{\hbar}\left(V-V_{\mathrm{eff}}-C\right) \operatorname{Im}\left[\Psi_{+}{ }^{*} \Psi_{-}\right],
$$

where $j_{ \pm}= \pm v \rho_{ \pm}$is the flux, defined in terms of the generalized trajectory velocities. For the constant velocity case, this reduces to Ref. 3 Eq. (13).

In implementing the above scheme numerically, we borrow from the successful constant-velocity algorithm as much as possible. The condition that over a duration of time, $t_{\text {shift }}$, each grid point should progress to the precise spot vacated by its nearest neighbor, implies a very nonuniform grid - with a higher density of points in areas where $v$ is lower, as is appropriate. Though by no means necessary, we find it convenient to compute all of the trajectories in advance. Actually, only the single trajectory $x_{1}^{+}(t)$ need be computed-starting at $x=x_{L}$, and propagating classically in accord with $V_{\text {eff }}$ to a bit past $x=x_{R}$. The other trajectories can then be obtained via $x_{k}^{+}(t)=x_{1}^{+}\left[t+(k-1) t_{\text {shift }}\right]$, and $x_{k}^{-}(t)=x_{k}^{+}(-t)$. The $x_{1}^{+}(t)$ values for each positive integer multiple of the CPWM time step, $\Delta$, are computed and stored during this preprocessing phase, as are the corresponding effective potential values $V_{\text {eff }}, V_{\text {eff }}^{\prime}$, and $V_{\text {eff }}^{\prime \prime}$ a technique that can in principle save much CPU time during the subsequent CPWM propagation phase. Note that by design, the trajectory version of Eq. (16) involves no explicit spatial differentiation of $\Psi_{ \pm}$; once again, the largest source of numerical error (and CPU effort) is interpolation. To ensure that the trajectory calculation does not contribute appreciably to the error, it is performed using a much smaller time step, $\delta=\Delta / 100$; even so, the CPU cost of the preprocessing phase is a trivial part of the whole cost. Also, numerical comparisons with $\delta=\Delta / 30$ and $\Delta / 300$ reveal no discernible difference in computed results.

Another difference from the constant-velocity scheme is the choice of initial conditions. Based on the discussion in Sec. IA we take $\Psi_{+}(t=0)$ to be the basic WKB solution, i.e.

$$
\Psi_{+}(x, 0)=\sqrt{v_{L} / v(x)} \exp \left[i s_{0}(x) / \hbar\right],
$$

where the initial action, $s_{0}(x)$, is also computed during the preprocessing phase (the initial reflected wave is of course still zero). The generalization of Eq. (7) is seemingly non-trivial:

$$
\begin{aligned}
\Psi_{ \pm k}(t+\Delta)= & \Psi_{ \pm k}(t) \\
& \exp \left\{\frac{i}{\hbar} \Delta\left[E-2 V_{\mathrm{eff}} \pm \frac{i}{4} \hbar v\left(\frac{V_{\mathrm{eff}}^{\prime}}{E-V_{\mathrm{eff}}}\right)\right]\right\} \\
& -\frac{i}{\hbar} \Delta\left(V-V_{\mathrm{eff}}-C\right)\left[\Psi_{ \pm k}(t)+\Psi_{\mp}\left(x_{k}^{ \pm}, t\right)\right]
\end{aligned}
$$

with all functions evaluated at $x_{k}^{ \pm}$. The rationale is the same as before however: the asymptotically vanishing coupling term in Eq. (16) (but for $\Psi$, rather than just $\Psi_{ \pm}$) is treated using forward Euler propagation; everything else is exponentiated as per Eq. (6).

The proceeding discussion is completely general, i.e. applies to any choice of $V_{\text {eff }}(x)$. However, for singlebarrier reactions (no intermediates), we advocate the following analytical ramp functional form, whose derivatives are also specified:

$$
\begin{aligned}
& V_{\text {eff }}(x)=\left(\frac{V_{R}-V_{L}}{2}\right)\left\{\tanh \left[\beta\left(x-x_{0}\right)\right]+1\right\} \\
& V_{\text {eff }}^{\prime}(x)=\left(\frac{V_{R}-V_{L}}{2}\right)\left\{\frac{\beta}{\cosh ^{2}\left[\beta\left(x-x_{0}\right)\right]}\right\} \\
& V_{\text {eff }}^{\prime \prime}(x)=-\left(V_{R}-V_{L}\right) \beta^{2}\left\{\frac{\tanh ^{2}\left[\beta\left(x-x_{0}\right)\right]}{\cosh ^{2}\left[\beta\left(x-x_{0}\right)\right]}\right\}
\end{aligned}
$$

As in Sec. IIIC $x_{0}$ should be in the middle of the $V(x)$ interaction region, and $1 / \beta$ comparable to the interaction region size.

\section{E. Summary and Numerical Recipes}

To summarize, the key numerical developments in this section have been: (1) use of trajectory grids that are coincident when $t$ is a multiple $t_{\text {shift }}$ (implying that $t_{\text {shift }}$ itself is a multiple of $\Delta$ ); (2) unitary plane wave (or exponential) propagation throughout the coordinate range; (3) third-order spline interpolation of $r_{ \pm}$and $s_{ \pm}$, to evaluate the coupling contribution to the time-evolution equations.

For asymptotically symmetric potentials, the recipe for numerical propagation of the constant velocity trajectory quantities is as follows:

- Move each grid point, $x_{k}^{ \pm}$, via $x_{k}^{ \pm}(t+\Delta)=x_{k}^{ \pm}(t) \pm$ $v \Delta$

- For exterior grid points outside the range of the opposite grid, update $\Psi_{ \pm k}$ via Eq. (6). 
- For interior grid points, update $\Psi_{ \pm k}$ via Eqs. (7), (8), and (9).

For asymptotically asymmetric potentials, the ramp trajectory method is preferred over the discontinuous trajectory method (Sec. IV). The ramp trajectories are computed and stored a priori, in a preprocessing step. The subsequent numerical propagation is as follows:

- For exterior grid points outside the range of the opposite grid, update $\Psi_{ \pm k}$ via the first line of Eq. (20).

- For interior grid points, update $\Psi_{ \pm k}$ via Eqs. (20), (8), and (9).

\section{RESULTS}

In this section, the three different numerical algorithms described in detail in Sec. III-i.e. the constant velocity, discontinuous constant velocity, and ramp trajectory versions of the non-wave-front $\mathrm{F}$ trajectory method - are applied to a variety of test applications, in order to assess them for robustness and numerical efficiency. In all cases, first-order forward Euler time-evolution is employed as discussed previously, leading to "artificially" small time steps, $\Delta$ (e.g. as compared to the fourth-order RungeKutta results of Ref. 3 ). The mass $m=2000$ a.u. is used in all applications.

A thorough and detailed convergence study was performed for each application as follows. First, given the small oscillations with $x$ that characterize the numerical $\rho_{ \pm}(x)$ solutions in the asymptotic regions, the usual determination of reflection and transmission probabilities as $P_{\text {refl }}=\left|\Psi_{-}\left(x_{L}\right)\right|^{2} ; P_{\text {trans }}=\left(v_{R} / v_{L}\right)\left|\Psi_{+}\left(x_{R}\right)\right|^{2}$ is not used. Instead, a small range of points near $x_{L / R}$ is consulted to characterize both mean values and uncertainties for these quantities. Reducing the oscillatory uncertainty to an acceptably small level is one of two conditions that must be satisfied to achieve convergence; the second is that variation of the mean $P_{\text {reff }}$ and $P_{\text {trans }}$ values with respect to all numerical parameters be acceptably small. The convergence parameters, in the cyclical order in which they are varied (keeping all others fixed) are as follows: maximum time, $t_{\max }$; grid spacing, $\Delta x$; time step, $\Delta$; grid boundaries, $x_{L / R}$. For each of the applications considered, tens of convergence calculations were performed, usually over multiple cycles.

\section{A. The Eckart Barrier}

The first application considered is the symmetric Eckart barrier, $, 44,45$

$$
V(x)=V_{0} \operatorname{sech}(\alpha x)^{2},
$$

with parameter values $V_{0}=400 \mathrm{~cm}^{-1}$, and $\alpha=3.0$ a.u, which for the present paper is labeled "Eckart A." This

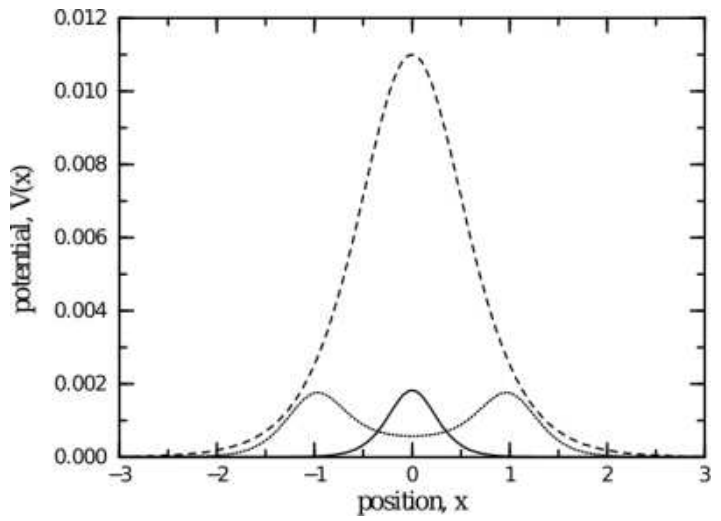

FIG. 2: Potential energy curves for several symmetric potential systems considered in this paper: Eckart A barrier (solid); Eckart B barrier (dashed); double barrier (dotted). All units are atomic units.

application is identical to the Eckart problem of Ref. $\underline{3}$, and is chosen here in part to compare with the previous results. A plot of this potential is presented in Fig. 2

A detailed convergence study was performed for the energy equal to the barrier peak, i.e. $E=V_{0}=400 \mathrm{~cm}^{-1} \approx$ 0.0018 hartree. This energy was chosen because it provides a good splitting of probability between $P_{\text {refl }}$ and $P_{\text {trans }}$. Of the three numerical methods considered, only the constant velocity method is applicable here-because both the discontinuous constant velocity and ramp trajectory methods reduce to the constant velocity method when the potential $V(x)$ is symmetric. In any event, the parameters that resulted from the convergence procedure described above, with $10^{-4}$ as the target accuracy for both $P_{\text {refl }}$ and $P_{\text {trans }}$, are presented in Table \ column 3 . These can be compared directly to the Ref. 3 calculation (computed to the same level of accuracy), presented in column 2 , as well as to the analytical values given in the last two rows.

The table indicates that the new algorithm requires substantially fewer grid points than the old - a testament to the efficacy of the new PWP and interpolation routines. However, the new algorithm requires a much smaller time step (more than $50 \times$ ), owing to the fact that the non-PWP time integrator is only first-order, rather than fourth-order. Presumably for the same reason, $\Delta$ was found to be the slowest parameter to converge. From Table I it is also clear that the computed $P_{\text {refl }}$ value is substantially more accurate than $P_{\text {trans }}$. Since only one of these two quantities is needed in practice (because their sum is unity), we performed a second convergence study for which $P_{\text {refl }}$ alone was converged to $10^{-4}$. The results, presented in Table \ column 4 , indicate a very substantial efficiency improvement, requiring a mere $N=13$ grid points and 0.88 seconds on a $2.60 \mathrm{GHz}$ Pentium CPU - as compared to $N=31$ and 5.0 seconds for column 2. Note that for both calculations, the oscillatory error is substantially smaller than the overall convergence errori.e. it would have been essentially just as accurate to use 
TABLE I: Convergence parameters for constant velocity trajectory method applied to several symmetric potential systems, with energies near or at the barrier height. All units are atomic units. Column 2: older algorithm used in Ref. 3. Columns 3-6: newer algorithm of Sec. IIA Convergence of quantities indicated is to $10^{-4}$ for Eckart A calculations, and $10^{-3}$ for others. Rows 7 and 8: computed reflection and transmission probabilities, including oscillatory uncertainties. Last two rows: exact reflection and transmission probabilities for Eckart systems.

\begin{tabular}{|c|c|c|c|c|c|}
\hline \multirow{3}{*}{$\begin{array}{c}\text { Quantity } \\
\text { and } \\
\text { symbol }\end{array}$} & \multicolumn{5}{|c|}{ Symmetric potential system } \\
\hline & \multicolumn{3}{|c|}{ Eckart A } & \multirow{2}{*}{$\begin{array}{l}\text { Eckart B } \\
P_{\text {refl }} \text { only }\end{array}$} & \multirow{2}{*}{$\begin{array}{c}\text { double Gaussian } \\
P_{\text {refl }} \text { and } P_{\text {trans }}\end{array}$} \\
\hline & Ref. 3 & $P_{\text {refl }}$ and $P_{\text {trans }}$ & $P_{\text {refl }}$ only & & \\
\hline grid size, $N$ & 31 & 20 & 13 & 25 & 20 \\
\hline left edge, $x_{L}$ & -3.0 & -2.0 & -2.0 & -3.0 & -3.0 \\
\hline right edge, $x_{R}$ & 3.0 & 2.0 & 1.5 & 2.1 & 2.5 \\
\hline grid spacing, $\Delta x$ & 0.20 & 0.211 & 0.292 & 0.213 & 0.289 \\
\hline time step, $\Delta$ & 10.0 & 0.156 & 0.324 & 0.046 & 0.612 \\
\hline $\max$ time, $t_{\max }$ & 10000 & 3899 & 4105 & 3204 & 41015 \\
\hline computed $P_{\text {refl }}$ & & $.28336 \pm .00004$ & $.28323 \pm .00010$ & $.4587 \pm .0002$ & $.7936 \pm .0002$ \\
\hline computed $P_{\text {trans }}$ & & $.71646 \pm .00005$ & $.71625 \pm .00005$ & $.5355 \pm .0003$ & $.20498 \pm .00001$ \\
\hline exact $P_{\text {refl }}$ & & 0.283358 & 0.283358 & 0.459605 & \\
\hline exact $P_{\text {trans }}$ & & 0.716642 & 0.716642 & 0.540395 & \\
\hline
\end{tabular}

$P_{\text {trans }}=\rho_{+}\left(x_{R}\right)$, etc.

Finally, column 5 of Table 1 presents results for the much broader and taller "Eckart B" barrier, i.e. with parameter values $V_{0}=0.011$ hartree $\left(\approx 2414 \mathrm{~cm}^{-1}\right)$, and $\alpha=1.364$ a.u. The Eckart B potential is also presented in Fig. 2. The goal here is to determine whether the new algorithm is robust across a wide range of system parameters, and to what extent the much larger-action Eckart B system requires additional computational effort. The Eckart B system is also used to analyze deep tunneling performance in Sec. IVB. Here, though, we consider only the barrier energy $E=V_{0}$. A comparison between columns 4 and 5 reveals that a larger grid and smaller time step are needed in the Eckart B case, as would be expected based on system parameters. However, the near order-of-magnitude time step reduction is far greater than expected-particular given that the Eckart B calculation is less accurate to one digit of precision. This is due to the convergence of computed error with decreasing $\Delta$, which, though initially fast, is found to become quite slow (essentially inverse scaling) beyond a certain threshold accuracy level. For Eckart B, this threshold appears to be around $3 \times 10^{-3}$. In any case, higher-order time evolution is expected to resolve this difficulty completely.

\section{B. The Deep Tunneling Regime}

To analyze the efficacy of the constant velocity method in the deep tunneling regime, the Eckart B problem was solved for a range of below-barrier energies, $E<V_{0}$. The large size of this barrier ensures that even energies relatively close to the top of the barrier-e.g., $E=0.8 V_{0}$ are characterized by quite small transmission probabilities, $P_{\text {trans. }}$ By $E=0.1 V_{0}$, the $P_{\text {trans }}$ values are extremely small (on the order of $10^{-9}$ ). Detailed convergence studies were performed for the above two energy values, as well as for the intermediate value $E=0.4 V_{0}$, in order to assess the method across the whole energetic range. The details are presented in Table II For simplicity, $x_{L}=x_{R}$ was presumed. The quantity $P_{\text {trans }}$ was converged to an absolute accuracy that varied substantially with $E$, as indicated in Table II row 6 . In relative accuracy terms, this translates to three-to-five significant digits for each computed $P_{\text {trans }}$ value, with the $E=0.4 V_{0}$ case substantially more accurate than the other two. In all cases, the convergence error is comparable to the actual error, obtained relative to the exact $P_{\text {trans }}$ values presented in row 8 . These findings are particularly satisfying for the extreme $E=0.1 V_{0}$ case, which is computed here to an absolute error of $10^{-12}$ or so.

The convergence trends with respect to decreasing $E$, as evident in Table III, are illuminating. Perhaps most striking is the fact that only a modest increase in coordinate range, $x_{L / R}$, is observed-completely unlike the usual situation for conventional quantum scattering methods (see below). A modest increase in grid size, $N$, arising primarily from a reduction in grid spacing, $\Delta x$, is nevertheless required. This is true, even though the wavelength increases with decreasing $E$, because the lower energy calculations must be performed to a substantially higher level of absolute accuracy. Comparing rows 1 and 6 , the convergence appears to be exponentially fast, with something like twelve extra points needed for each additional digit of accuracy. Relative accuracy also plays a role in this regard, however, e.g. the $0.8 V_{0}$ calculation done to the same absolute accuracy as the $0.4 V_{0}$ calculation is found (not presented in Table II) to require a greater number of grid points than the latter.

There is a very substantial growth of $t_{\max }$ with decreasing energy, owing to the fact that the trajectories move much more slowly. However, for the same reason, there is also a substantial increase in the time step size, $\Delta$, so that the growth in the total number of time steps (and CPU effort) is much more modest. The above comments 
TABLE II: Convergence parameters for constant velocity trajectory method applied to Eckart B system over a wide range of below-barrier energies, $E<V_{0}$. All units are atomic units. Convergence is of transmission probability, $P_{\text {trans }}$, only, to accuracy level indicated in row 6 . Computed $P_{\text {trans }}$ values including oscillatory uncertainty are listed in row 7 , with exact values given in row 8.

\begin{tabular}{cccc}
\hline \hline Quantity & \multicolumn{3}{c}{ Energy $E$ as fraction of barrier height $V_{0}}$. \\
\cline { 2 - 4 } and symbol & $0.8 V_{0}$ & $0.4 V_{0}$ & $0.1 V_{0}$ \\
\hline grid size, $N$ & 33 & 88 & 133 \\
grid edges, $x_{L / R}$ & $\mp 3.0$ & $\mp 4.0$ & $\mp 4.0$ \\
grid spacing, $\Delta x$ & 0.188 & 0.092 & 0.061 \\
time step, $\Delta$ & 0.126 & 0.097 & 1.156 \\
max time, $t_{\max }$ & 4424 & 46424 & 462285 \\
target accuracy for $P_{\text {trans }}$ & $0.02(-2)$ & $0.0005(-5)$ & $0.01(-10)$ \\
computed $P_{\text {trans }}$ & $4.444 \pm .020(-2)$ & $1.5588 \pm .0004(-5)$ & $9.920 \pm .002(-10)$ \\
exact $P_{\text {trans }}$ & $4.462(-2)$ & $1.5594(-5)$ & $9.920(-10)$ \\
\hline \hline
\end{tabular}

apply for calculations at comparable relative accuracies, e.g. Table II columns 2 and 4 . The intermediate calculation (column 3), done to a substantially higher relative accuracy than the others, is found to be in the regime of slow convergence with respect to $\Delta$ (Sec. IVA) and therefore manifests a much smaller converged value for this parameter.

The extreme $E=0.1 V_{0}$ calculation represents a very challenging test case for conventional quantum dynamics methods. For comparative purposes, an optimized sincDVR calculation $34,35,46$ with quartic absorbing boundary conditions was also performed. Such methods always increase the coordinate range beyond the interaction region - to a degree proportional to the wavelength, and thus very substantial at low energies. For instance, $x_{L / R}=\mp 40.0$ a.u. for the DVR calculation, a full order of magnitude larger than for the CPWM calculation. The resultant grid size, $N=700$, was also much largerparticularly given that CPU effort scales as $N^{3}$ for DVR methods, but only as $N$ for the present approach.

The above discrepancies are magnified even more for shorter, narrower barriers than Eckart B. In this context, deep tunneling is observed only when $E$ is a tiny fraction of $V_{0}$. For the Eckart A problem with $E=10^{-4} V_{0}$, for instance, attempts to perform a converged sinc-DVR calculation proved unsuccessful, owing to the enormous coordinate range required. Instead, an approximate, semiclassical tunneling calculation was performed, giving rise to the prediction $P_{\text {trans }}=3.82 \times 10^{-3}$. In fact, this is more than $100 \times$ larger than the actual value, $P_{\text {trans }}=2.851 \times 10^{-5}$. These examples serve to underscore the difficulty of computing accurate deep tunneling transmission probabilities using conventional methods, which may be greatly alleviated with the present bipolar CPWM approach.

\section{The Uphill Ramp}

The first asympotically asymmetric system considered here is the continuous "uphill ramp," 47 defined via Eq. (20) and the parameter values $V_{L}=0, V_{R}=$

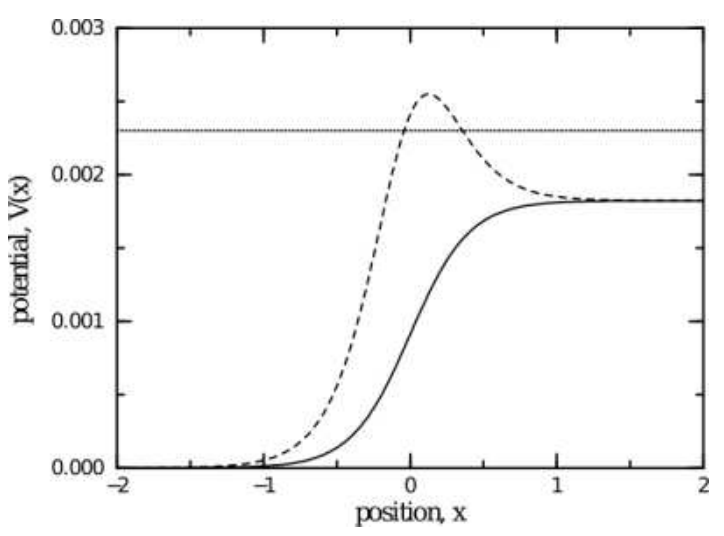

FIG. 3: Potential energy curves for two asymmetric potential systems considered in this paper: uphill ramp (solid); barrier ramp (dashed). Dotted line indicates energy value, $E=0.0023$, used for both systems. All units are atomic units. For the ramp trajectory calculations for both systems, the trajectory potential, $V_{\text {eff }}(x)$, is the solid curve.

$400 \mathrm{~cm}^{-1} \approx 0.0018, x_{0}=0$, and $\beta=2.5$ a.u. Thus, $V(x)=V_{\text {eff }}(x)$, so that the ramp trajectories are themselves classical trajectories. The energy $E=0.0023$ a.u $\approx 500 \mathrm{~cm}^{-1}$ is deliberately chosen to be only moderately larger than $V_{R}$, to accentuate the asymmetric difference between $v_{L}$ and $v_{R}$. A plot is presented in Fig. 3. The system and energy parameters are identical to those considered in Ref. 3, and are also comparable to those of the Eckart A calculation in Sec. IV A enabling a fairly direct comparison. Detailed convergence studies were performed for both the discontinuous constant velocity and ramp trajectory methods, as presented in Table III. Both $P_{\text {refl }}$ and $P_{\text {trans }}$ were converged, with the former to a higher level of accuracy, appropriate to the different scales of the two quantities. Note that this is the first example considered for which $P_{\text {trans }} \gg P_{\text {refl }}$.

The discontinuous constant velocity case is considered first, i.e. Table III column 2. As compared with the Eckart A calculation in Table 1 column 3, the most compelling difference is that a much higher density of grid points is needed ( $N=80$ vs. $N=20$ ), even though the 
TABLE III: Convergence parameters for discontinuous constant velocity and ramp trajectory methods, applied to two asymmetric potential systems with energy $E=0.0023$ a.u $\approx 500 \mathrm{~cm}^{-1}$ slightly larger than asymptotic potential difference $\left(V_{R}-V_{L}\right)=400 \mathrm{~cm}^{-1}$. All units are atomic units. Columns 2 and 3: uphill ramp system. Columns 4 and 5 barrier ramp system. Convergence is of both $P_{\text {refl }}$ and $P_{\text {trans }}$ to accuracy levels indicated in rows 7 and 8 . Last two rows: computed reflection and transmission probabilities, including oscillatory uncertainties.

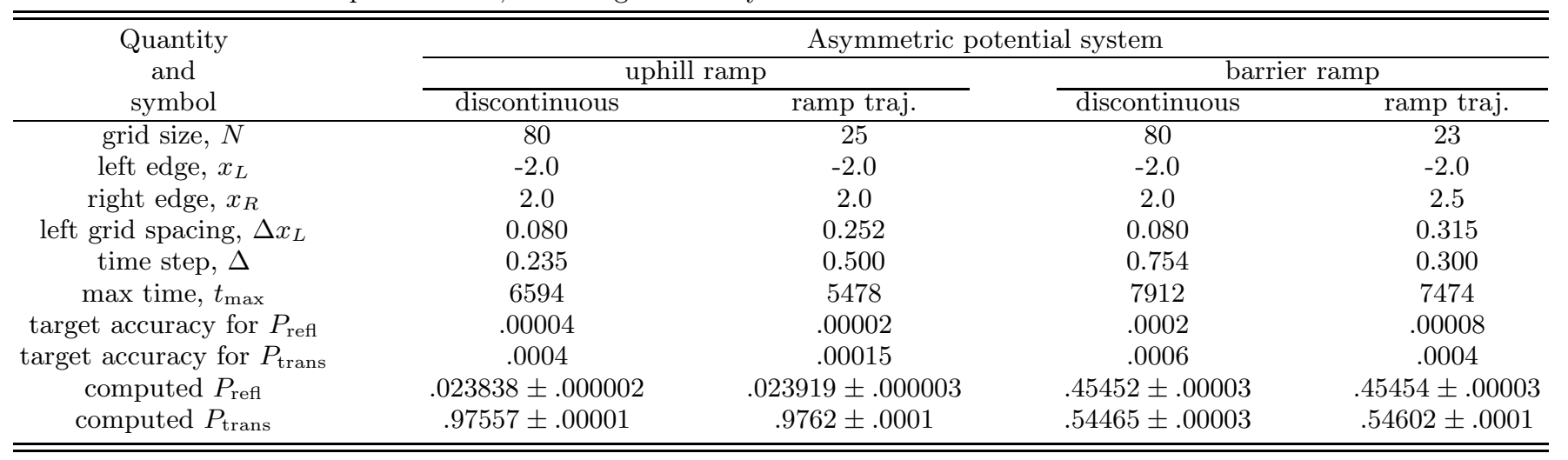

final computed accuracy is somewhat lower. This is most likely attributable to the use of extrapolation (Sec. III C) in the middle of the interaction region where the coupling is greatest. One indication is the fact that the asymptotic oscillations are much smaller than for Eckart A, and not a contributing factor to the total error. Another significant difference is found in the parameter $t_{\max }$, which is larger in the uphill ramp case, probably owing to the fact that product trajectories are substantially slower than for Eckart A. Perhaps for the same reason, $\Delta$ is also somewhat larger for the uphill ramp.

The ramp trajectory convergence parameters are shown in Table III column 3 . The $N=25$ grid size is now greatly reduced in comparison to column 2, almost to the Eckart A level, again suggesting that the primary source of error in the discontinuous case is extrapolation. The small increase in $N$ relative to Eckart A may be due to the fact that grid spacing in product and reactant regions are not independently adjustable (which may also be a contributing factor in the discontinuous calculation). Another key difference between the two asymmetric calculations is that $t_{\max }$ is substantially reduced in the ramp trajectory case - most likely reflecting the fact that the initial semiclassical approximation $\Psi_{+}(x, 0)$ is much closer to the actual solution (Sec. IIID). Nevertheless, the time step $\Delta$ is substantially larger. Taken together, all of the above findings imply that CPU effort for the ramp trajectory calculation is greatly reduced in comparison to the discontinous calculation.

\section{The Barrier Ramp}

The barrier ramp system (Fig. 3) is the first asymmetric reaction with a barrier to be treated using CPWM methods. Serving as representative of a generic reaction profile, moreover, it is an extremely important benchmark system. This is especially true for the ramp trajec- tory method, because $V_{\text {eff }}(x)$, being monotonic, can no longer be equal to $V(x)$, which might in principle affect computational efficiency. To construct a suitable barrier ramp potential, we simply add an Eckart barrier and an uphill ramp together. The Eckart parameters [Eq. (21)] are taken to be $V_{0}=0.0015$ a.u. and $\alpha=2.5$ a.u, whereas the uphill ramp is identical to that of Sec. IV C. The ramp trajectories are also identical to Sec. IV C, i.e. $V_{\text {eff }}(x)$ is the same as before. The energy $E=0.0023$ a.u is also the same as in Sec. IVC, as are the asymptotic trajectory velocities, $v_{L / R}$.

The discontinuous and ramp trajectory convergence parameters are shown in Table III columns 4 and 5, respectively. In both cases the grid parameters are nearly identical to the corresponding uphill ramp calculations (columns 2 and 3)-implying that the presence of the barrier introduces no numerical difficulties for either method. The $t_{\max }$ values are somewhat larger, undoubtedly owing to the fact that the barrier ramp case has much greater reflection. 2.3 For the discontinuous calculation, $\Delta$ is lower than the uphill ramp value, perhaps because the absolute accuracy of the computed $P_{\text {refl }}$ is much less. This situation is reversed for the ramp trajectory calculation, for which a somewhat smaller $\Delta$ is required. The reason may have to do with the fact that $V_{\text {eff }}(x) \neq V(x)$, giving rise to larger coupling in Eq. (16).

\section{E. The Double Barrier}

The last case to be considered is a system with reaction intermediates. In Ref. 3, a double-Gaussian barrier was employed. Here however, we wish to have more flexibility in the functional form, in order that a wider range of reaction profiles may be considered. To achieve this, a sum of two barrier ramps is used, centered at the transition states on either side of the reaction intermediate. Though symmetry is not required, for simplicity we adopt 
the symmetric form here as follows:

$$
\begin{aligned}
V(x)= & V_{0}\left\{\operatorname{sech}\left[\alpha\left(x+x_{0}\right)\right]^{2}+\operatorname{sech}\left[\alpha\left(x-x_{0}\right)\right]^{2}\right\}+ \\
& \left(V_{\Delta} / 2\right)\left\{\tanh \left[\beta\left(x+x_{0}\right)\right]-\tanh \left[\beta\left(x-x_{0}\right)\right]\right\}
\end{aligned}
$$

The parameters $V_{0}=0.0015$ a.u, $x_{0}=1.0, \alpha=\beta=2.5$ a.u., and $V_{\Delta}=0.0005$ a.u. are comparable to those used for the barrier ramp and Eckart A systems. The energy, $E=0.0014$ a.u, lies slightly below the barrier peak of around 0.00175 a.u. A plot is presented in Fig. 2

Although this system would be a good candidate for a double ramp trajectory scheme (Sec. IIID), the constant velocity trajectory algorithm is used here. Convergence for both $P_{\text {refl }}$ and $P_{\text {trans }}$ to $10^{-3}$ is indicated in Table I column 6 . This accuracy is somewhat lower than for Eckart A (column 3), to avoid slow $\Delta$ convergence (Sec. IVA). The coordinate range is larger than for Eckart A, but the grid density is actually somewhat smaller (despite the fact that the potential has more features), such that the $N=20$ grid size is the same for both. The convergence time $t_{\max }$ is much larger than for Eckart A, as is expected for a system with tunneling and reaction intermediates $\frac{2,3}{2}$ In other respects, the calculation is similar to, and no more difficult than, the other calculations without reaction intermediates.

\section{CONCLUSION}

Based on the results as presented above, we conclude that all of the primary goals of this paper have been achieved. In particular, the robustness of the methods are demonstrated by the very broad range of test cases considered. These may be definitively regarded as "representative" of many other 1 DOF calculations (not described here) that were also performed. The efficiency of the methods is also established, in that most of the calculations that do not involve deep tunneling or reaction intermediates require no more than about one second of CPU time. Finally, the accuracy levels that can be achieved have been greatly expanded beyond previous limits, e.g., to twelve-to-thirteen digits past the decimal in the extreme deep tunneling case (Table II column 4) — probably the highest accuracy ever achieved in a trajectory-based quantum calculation. Although 1 DOF systems are usually regarded as trivially soluble, these can nevertheless pose severe numerical difficulties when extremely deep tunneling is involved; it is therefore particularly encouraging to see that the new methods work well in both this limit, and for more typical molecular situations.

Indeed, there do not appear to be any 1 DOF applications for which the present algorithms would not work well. This is of the utmost importance for the main motivation of the present work, i.e. creating a parameterfree (except for convergence parameters) "black-box" quantum dynamics code for solving any 1 DOF stationary scattering problem. A single universal algorithm is sought, although three specific implementations were considered in this paper: (1) constant velocity ; (2) discontinuous constant velocity; (3) ramp trajectory. As both (2) and (3) reduce to (1) in the asymptotically symmetric potential case, the only real choice is between (2) and (3). For reasons elucidated in Sec. IV] (3) is clearly the better choice, both with respect to numerical efficiency and robustness. As evidence of the latter, about the same number of grid points is required for systems with similar parameters, regardless of whether the potential is asymmetric, or has one or more barriers (compare Table I columns 3 and 6 with Table III columns 3 and $5)$.

The ramp trajectory scheme, (3), is therefore proposed for the universal purpose described above. One can imagine a straightforward procedure to automatize the selection of suitable ramp parameters for any given potential without reaction intermediates. Such a procedure would presumably be easily generalized to multiple ramp trajectories in the case of reaction intermediates, thus maintaining "black-boxness" for all cases. On the other hand, even a single ramp is seen to perform very well for multiple barrier systems [as in Sec. IVE, due perhaps to the smoothness of $V_{\text {eff }}(x)$ ], so that a single ramp may be all that is ever actually needed in practice. In fact an earlier preliminary investigation (without the numerical refinements of Sec. III) obtained slightly faster convergence and smoother field functions for single vs. double ramp calculations. Note that the dynamical equations derived in Sec. IIID are completely general, and could in principle be used to explore other candidate functional forms for $V_{\text {eff }}(x)$.

In any case, well-documented stand-alone fortran codes for all three algorithms considered here are available from the author on demand - which, in the case of (3) at least, will also incorporate future developments. Note that the weakest algorithmic aspect at present is the first-order forward time integration of the coupling term in the dynamical equations - leading to slow $\Delta$ convergence beyond a certain point, and much smaller typical $\Delta$ values than in Ref. 3 (10 a.u.), which used standard fourth-order Runge-Kutta integration. The first order of business is therefore to incorporate a similar timeintegrator into algorithm (3) - or perhaps something even more advanced, such as the time-adaptive Cash-Carp method, $\stackrel{42}{=}$ or multistep Adams-Bashforth method, $\underline{41}$ Preliminary investigations indicate that this will lead to far greater time step sizes even than those used in Ref. 3 e.g., $\Delta=100$ a.u. or more. If not the case already, such a modification would result in the most accurate and efficient quantum dynamics codes available for 1 DOF systems.

Of course, the primary challenge for future development is multidimensional systems. As discussed in Sec. I. the theoretical developments are already in place, both for stationary state and wavepacket scattering dynamics. These will be presented in detail in future publications, but a brief outline may be found in the Summary 
and Conclusions of Ref. 3. For the multidimensional stationary state method, numerical instability of the sort described here has proven to be an issue. Accordingly, new multidimensional algorithms will be developed that incorporate the modifications of the present work. In addition to (hopefully) providing numerical stability, the reduced number of grid points der DOF that characterizes the present approach should lead to far greater reductions in CPU effort for applications with more DOFs. For wavepacket dynamics, it turns out that more conventional Bohmian trajectories are appropriate (defined via $\left.m v_{ \pm}=s_{ \pm}^{\prime}\right)$. In anticipation of this, we have analyzed $s_{ \pm}^{\prime}$ curves for the stationary solutions obtained here, and found: (1) smooth, but not always monotonic, behavior; (2) asymptotic agreement with trajectories used here for all but the $\Psi_{-}(x \rightarrow \infty)$ asympotes; (3) exactly opposite trajectories in the $\Psi_{-}(x \rightarrow \infty)$ asymptote. The last observation, though curious, may not be significant, as $r_{-}(x)$ also vanishes in the same limit.

\section{Acknowledgments}

This work was supported by an award from The Welch Foundation (D-1523). Corey Trahan is acknowledged for performing some of the preliminary numerical investigations. Jason McAfee is also acknowledged for his aid in converting this manuscript to an electronic format suitable for the arXiv preprint server.
* Electronic address: Bill.Poirier@ttu.edu

1 B. Poirier, J. Chem. Phys. 121, 4501 (2004).

2 C. Trahan and B. Poirier, J. Chem. Phys. 124, 034115 (2006).

3 C. Trahan and B. Poirier, J. Chem. Phys. 124, 034116 (2006).

4 D. Babyuk and R. E. Wyatt, J. Chem. Phys. 121, 9230 (2004).

5 R. E. Wyatt, Quantum Dynamics with Trajectories: Introduction to Quantum Hydrodynamics (Springer, New York, 2005).

${ }^{6}$ C. L. Lopreore and R. E. Wyatt, Phys. Rev. Lett. 82, 5190 (1999).

7 F. S. Mayor, A. Askar, and H. A. Rabitz, J. Chem. Phys. 111, 2423 (1999).

8 R. E. Wyatt, Chem. Phys. Lett. 313, 189 (1999).

9 D. V. Shalashilin and M. S. Child, J. Chem. Phys. 113, 10028 (2000).

10 R. E. Wyatt and E. R. Bittner, J. Chem. Phys. 113, 8898 (2001).

11 R. E. Wyatt and K. Na, Phys. Rev. E 65, 016702 (2001).

12 I. Burghardt and L. S. Cederbaum, J. Chem. Phys. 115, 10312 (2001).

13 E. R. Bittner, J. B. Maddox, and I. Burghardt, Int. J. Quantum Chem. 89, 313 (2002).

14 K. H. Hughes and R. E. Wyatt, Phys. Chem. Chem. Phys. 5, 3905 (2003).

15 E. Madelung, Z. Phys. 40, 322 (1926).

16 J. H. van Vleck, Proc. Natl. Acad. Sci. U.S.A. 14, 178 (1928).

17 D. Bohm, Phys. Rev. 85, 166 (1952).

18 D. Bohm, Phys. Rev. 85, 180 (1952).

19 T. Takabayasi, Prog. Theor. Phys. 11, 341 (1954).

20 P. R. Holland, The Quantum Theory of Motion (Cambridge University Press, Cambridge, 1993).

21 D. Frenkel and B. Smit, Understanding Molecular Simulations (Academic, New York, 2002).

22 C. J. Trahan and R. E. Wyatt, J. Chem. Phys. 118, 4784 (2003).

23 B. K. Kendrick, J. Chem. Phys. 119, 5805 (2003).

${ }^{24}$ D. K. Pauler and B. K. Kendrick, J. Chem. Phys. 120, 603 (2004).

25 J. Heading, An Introduction to Phase-integral Methods
(Methuen, London, 1962).

26 N. Fröman and P. O. Fröman, JWKB Approximation (North-Holland, Amsterdam, 1965).

27 M. V. Berry and K. V. Mount, Rep. Prog. Phys. 35, 315 (1972).

28 J. C. Tully, J. Chem. Phys. 55, 562 (1971).

29 M. D. Wheeler, S. M. Newman, A. J. Orr-Ewing, and M. N. R. Ashfold, JJ. Chem. Soc. Faraday Trans. 94, 337 (1998).

30 see for example, Int. J. Quant. Chem. 14, (1978), special issue.

31 W. P. Reinhardt, Ann. Rev. Phys. Chem. 33, 223 (1982).

${ }^{32}$ V. Ryaboy, N. Moiseyev, V. A. Mandelshtam, and H. S. Taylor, J. Chem. Phys. 101, 5677 (1994).

33 G. Jolicard and E. J. Austin, Chem. Phys. Lett. 121, 106 (1985).

34 T. Seideman and W. H. Miller, J. Chem. Phys. 96, 4412 (1992).

35 T. Seideman and W. H. Miller, J. Chem. Phys. 97, 2499 (1992).

36 U. V. Riss and H.-D. Meyer, J. Phys. B: At. Mol. Phys. 26, 4503 (1993).

37 B. Poirier and T. Carrington, Jr., J. Chem. Phys. 119, 77 (2003).

38 B. Poirier and T. Carrington, Jr., J. Chem. Phys. 118, 17 (2003).

39 J. G. Muga, J. P. Palao, B. Navarro, and I. L. Egusquiza, Phys. Rep. 395, 357 (2004).

40 B. Fornberg, Math. Comp. 51, 699 (1988).

41 G. M. Phillips and P. J. Taylor, Theory and Applications of Numerical Analysis (Academic Press, New York, 1996).

42 W. H. Press et al, in Numerical Recipes, 1st ed. (Cambridge University Press, Cambridge, England, 1989).

43 J. R. Taylor, Scattering Theory (John Wiley \& Sons, Inc., New York, NY, 1972).

44 C. Eckart, Phys. Rev. 35, 1303 (1930).

45 Z. Ahmed, Phys. Rev. A 47, 4761 (1993).

46 D. T. Colbert and W. H. Miller, J. Chem. Phys. 96, 1982 (1992).

47 S. Flugge, Practical Quantum Mechanics (Springer-Verlag, New York, 1971), Vol. 1. 